\title{
Thiol-Capped Germanium Nanocrystals: Preparation and Evidence for Quantum Size Effects
}

Elayaraja Muthuswamy, Jing Zhao, Katayoun Tabatabaei, Marlene M. Amador, Michael A. Holmes, Frank E. Osterloh, and Susan M. Kauzlarich*

Department of Chemistry, University of California, One Shields Avenue, Davis, California 95616, United States

Supporting Information

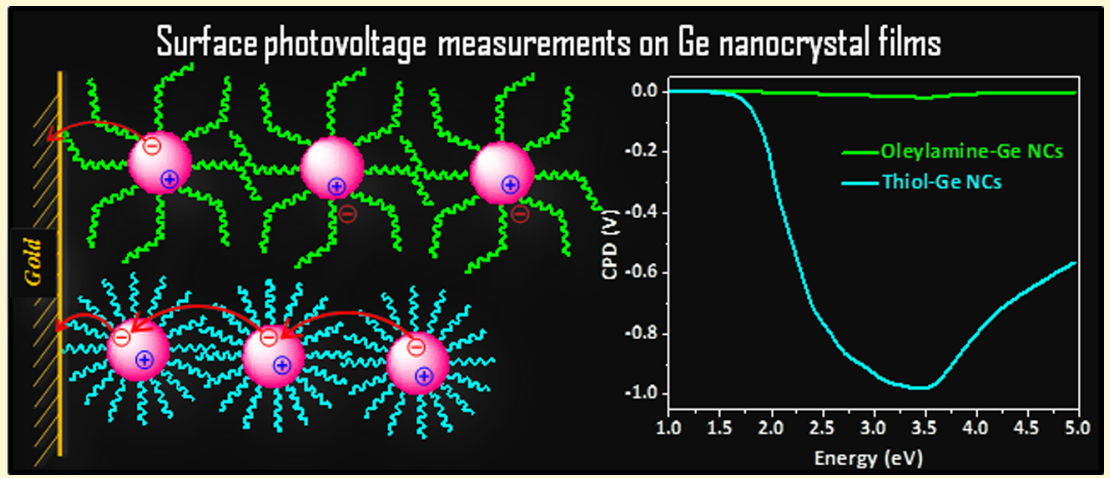

ABSTRACT: Applications of Ge nanocrystals (NCs) are limited by the stability and air reactivity of the Ge surface. In order to promote stability and increase the diversity of ligand functionalization of Ge NCs, the preparation of thiol-passivated Ge NCs via a ligand exchange process was investigated. Herein a successful replacement of oleylamine ligands on the surface of Ge NCs with dodecanethiol is reported. The successful ligand exchange was monitored by FTIR and NMR spectroscopy and it was found that dodecanethiol provided a better surface coverage, leading to stable Ge NC dispersions. Dodecanethiol capping also enabled band gap determination of the NCs by surface photovoltage (SPV) spectroscopy. The SPV measurements indicated an efficient charge separation in the ligand-exchanged Ge NCs. On the other hand, oleylamine-terminated Ge NCs of similar sizes exhibited a very small photovoltage, indicating a poorly passivated surface.

\section{INTRODUCTION}

Tunable properties can be achieved in nanoscale semiconductors due to quantum confinement effects via careful control of their size, shape, composition, and surface characteristics. Semiconductor nanocrystals (NCs) are currently explored for commercial applications and are promoted as next-generation materials for applications related to energy, ${ }^{1}$ lighting, ${ }^{2}$ biotechnology, and medicine. ${ }^{3}$ Group IV ( $\mathrm{Si}, \mathrm{Ge}$ ) NCs are of interest due to their relative nontoxicity and higher abundance in the earth's crust. ${ }^{4,5}$ Solution-based approaches for colloidal preparation offer a convenient approach for controlling size/morphology and surface modifications. However, challenges in colloidal preparation of $\mathrm{Si}$ and $\mathrm{Ge}$ with respect to size and yield have resulted in limited understanding of their size- and shape-dependent properties.

Ge has a number of characteristics that make it suitable for applications in solar energy conversion and electronics. It has a small band gap of $0.67 \mathrm{eV}$ (at $300 \mathrm{~K}$ ), superior absorption coefficient, and charge transport properties compared with silicon. ${ }^{6}$ Quantum confinement effects are activated in larger Ge NCs relative to Si due to the larger excitonic Bohr radius of $\mathrm{Ge}$ (24.3 vs $4.9 \mathrm{~nm}){ }^{7}$ As a thin film, Ge has been used in high efficiency tandem solar cells. ${ }^{8}$ There has been a growing interest in evaluating its properties at the nanoscale, and a variety of solution-based strategies have been reported for colloidal preparation of Ge NCs. Among them, the reduction of germanium iodides was the most promising for the control of size with high yield. ${ }^{9-12}$ The method was also shown to be adaptable for the preparation of alloyed Ge NCs. ${ }^{13}$ Utilizing that approach, we recently reported a reliable and robust microwave synthesis to prepare oleylamine-capped Ge NCs with size control in the range of $3-11 \mathrm{~nm} .{ }^{14}$ However, their instability as a dispersion when exposed to ambient conditions over a period of time and nonemissive characteristics in the expected near-IR region implies that these oleylamine-capped Ge NCs suffer from incomplete surface passivation.

Surface ligands play a critical role in determining the properties of NCs. While their insulating characteristics are considered detrimental for the construction of a NC-based device, their presence is beneficial in a number of other ways. In addition to controlling growth during the synthesis, they shield the NC surface against oxidation, offer stability against aggregation in solvents, ${ }^{15}$ and can provide a surface free of

Received: December 25, 2013

Revised: February 21, 2014

Published: February 26, 2014 
trap states, resulting in efficient emission characteristics. Amines, carboxylic acids, phosphines, thiols, and alkenes of differing chain lengths are typically used as coordinating ligands for NCs. A number of ligands have been explored for Ge NC surface capping and reported in the literature.

The earliest reports on colloidal synthesis of Ge NCs date back to 1993 wherein the reduction of $\mathrm{GeCl}_{4}$ by sodium metal and lithium naphthalide was investigated. ${ }^{16,17}$ Heath and coworkers reported the presence of hydrocarbons, oxygen, and chlorine on the surface of $\mathrm{Ge}$ crystallites. ${ }^{16}$ Weller and coworkers presumed termination of the surface by $\mathrm{CH}_{3} \mathrm{Si}$ radicals, generated by the addition of $\left(\mathrm{CH}_{3}\right)_{3} \mathrm{SiCl}^{17} \mathrm{Zintl}$ salts together with $\mathrm{GeCl}_{4}{ }^{18,19}$ or $\mathrm{NH}_{4} \mathrm{Br}^{20}$ were first utilized by our group for $\mathrm{Ge} \mathrm{NC}$ synthesis. The reaction with $\mathrm{GeCl}_{4}$ yielded chlorineterminated NCs, which were subsequently reacted with different Li-alkyls to passivate their surfaces with alkyl groups. In a subsequent work, covalent attachment of a range of functional groups such as acetals, alcohols, esters, and polymers was also achieved. ${ }^{21}$ Chlorine/hydrogen-terminated NCs have been prepared under plasma conditions by dissociating a mixture of $\mathrm{GeCl}_{4}$ and $\mathrm{H}_{2}{ }^{22}$

Alkyl phosphines [trioctylphosphine (TOP), tributylphosphine] as solvents and capping ligands have been utilized in $\mathrm{Ge}$ $\mathrm{NC}$ synthesis, but conclusive evidence for a $\mathrm{Ge}-\mathrm{P}$ bond by FTIR was not observed. ${ }^{9}$ However, the spectrum obtained for the NCs was consistent with neat TOP. Oleylamine was first used as a capping ligand for Ge NCs by Gerung and coworkers. $^{23}$ Oleic acid was also used as a capping ligand along with oleylamine in the preparation of highly crystalline Ge NCs. ${ }^{11}$ Hydrogermylation reactions with multiple carboncarbon bond containing organic compounds are a common method of modifying the hydrogen termination into a more stable $\mathrm{Ge}-\mathrm{C}$ bond at the surface. 1-Octadecene ${ }^{10,12}$ was utilized to prepare Ge NCs dispersible in nonpolar solvents, and allylamine ${ }^{24}$ was used to prepare Ge NCs dispersible in water.

A recent review by Schaak and co-workers addresses the developments made in colloidal preparation of Ge NCs. ${ }^{5}$ Only a few reports discuss size-dependent emission properties for $\mathrm{Ge}$ NCs. Size-dependent near-IR emission has been reported, ${ }^{10,12,25}$ as well as emission in the visible region ${ }^{19,20,23,24,26,27}$ for similarly sized Ge NCs. While progress has been made through these attempts toward creating a synthetic method that allows control of Ge NC size, there is still a great deal of inconsistency, and more effort is required to understand their size-dependent properties. Superior corrosion and oxidation resistance and better stability have been observed in the case of thiol-capped Ge nanowires, indicating that thiols should provide a stable passivating ligand for Ge NCs as well. ${ }^{28-30}$ While partial ligand exchange by thiols has been shown previously, ${ }^{31}$ thiols have not been explored in detail for complete passivation of a Ge NC surface. In this work, we have utilized alkanethiols (dodecanethiol, octadecanethiol) as a capping ligand and have studied the properties of thiol-capped Ge NCs via surface photovoltage (SPV) spectroscopy.

\section{EXPERIMENTAL SECTION}

Chemicals. Oleylamine ( $>40 \%)$ was purchased from TCI America and was used after degassing under vacuum at $100{ }^{\circ} \mathrm{C}$. Dodecanethiol (98\%) was purchased from Sigma Aldrich and was degassed at $100{ }^{\circ} \mathrm{C}$, prior to use. Hydrazine (95\%) and octadecanethiol (98\%) were purchased from Sigma Aldrich and used as received. Germanium(II) iodide was received from Prof. Richard Blair's laboratory (University of South Florida) and was characterized by powder X-ray diffraction (PXRD) to be phase-pure. ${ }^{32}$ Germanium(IV) iodide (99.999\% Ge) was purchased from STREM Chemicals. Methanol, hexane, toluene, and acetonitrile were purchased from Fisher Scientific, purified using a solvent purification system, and stored in a glovebox under argon.

Synthesis. Ge NC synthesis was carried out in a CEM microwave reactor (Discover SP). The microwave reactor is calibrated by a CEM technician periodically, and the reported temperatures are expected to be within $\pm 5{ }^{\circ} \mathrm{C}$. In an argon-filled glovebox, $0.4 \mathrm{mmol}$ of $\mathrm{GeI}_{2}$ was placed in a $35 \mathrm{~mL}$ microwave tube along with $\sim 8 \mathrm{~mL}$ of oleylamine. The microwave tube was sonicated to completely dissolve the $\mathrm{GeI}_{2}$. The dissolution resulted in a pale yellowish solution and the system was heated to $210{ }^{\circ} \mathrm{C}$ in the microwave reactor. The reaction was typically carried out for $60 \mathrm{~min}$. Reduction occured during the reaction and resulted in a brownish system, indicating the formation of $\mathrm{Ge}$ NCs. The contents of the microwave tube were transferred into a centrifuge tube. Washing with $2-3 \mathrm{~mL}$ of toluene helps to transfer all of the NCs, and excess methanol $(30-35 \mathrm{~mL})$ was added to precipitate the NCs. Centrifugation $(8000 \mathrm{rpm})$ for about $10-15 \mathrm{~min}$ resulted in complete precipitation of the NCs. The colorless supernatant was discarded and the oleylamine-terminated Ge NCs were dispersed in about 5-6 $\mathrm{mL}$ of toluene or hexane and stored in a vial under argon until required for measurements. To prepare larger sized Ge NCs, a predetermined quantity of $\mathrm{GeI}_{4}$ was added to the precursor and the reaction was carried out under identical conditions as reported in our previous publication. ${ }^{14}$

About $5.0 \mathrm{~mL}$ of the Ge NC dispersion in toluene was taken along with $5.0 \mathrm{~mL}$ of $5.0 \mathrm{M}$ hydrazine solution in acetonitrile in a glass vial. The system was stirred under an inert atmosphere for $60 \mathrm{~min}$ at room temperature, during which oleylamine was removed from the surface of the Ge NCs. The contents of the vial were centrifuged to isolate the ligand-stripped Ge NCs. The NCs were then washed with about 5-10 mL of toluene to remove any oleylamine present in the system. The removal of the oleylamine from the $\mathrm{NC}$ surface was qualitatively indicated by the loss of dispersibility of Ge NCs in toluene or hexane.

Thiol capping was carried out by treating the Ge NCs obtained after hydrazine treatment with about $10 \mathrm{~mL}$ of dodecanethiol. The system was heated in a microwave reactor at $150{ }^{\circ} \mathrm{C}$ for $1 \mathrm{~h}$, resulting in complete dispersion of the NCs in dodecanethiol. The binding of thiol to Ge was qualitatively confirmed by the ease with which the NCs disperse in dodecanethiol. Isolation of thiol-passivated Ge NCs is carried out by the addition of $10 \mathrm{~mL}$ of toluene and $25 \mathrm{~mL}$ of methanol to the Ge NC dispersion in dodecanethiol and subsequent centrifugation $(8000 \mathrm{rpm})$ for about $10-15 \mathrm{~min}$. The colorless supernatant was discarded, and the nanoparticles that settled at the bottom of the centrifuge tube were dispersed in about 5-6 mL of toluene or hexane. Further purification was carried out by precipitating the Ge NCs via centrifugation with excess methanol. The nanoparticles were stored as a dispersion in toluene or hexane in a glovebox.

Characterization. X-ray diffraction (XRD) patterns were collected by drop-casting the dispersion on a quartz substrate. Solvent

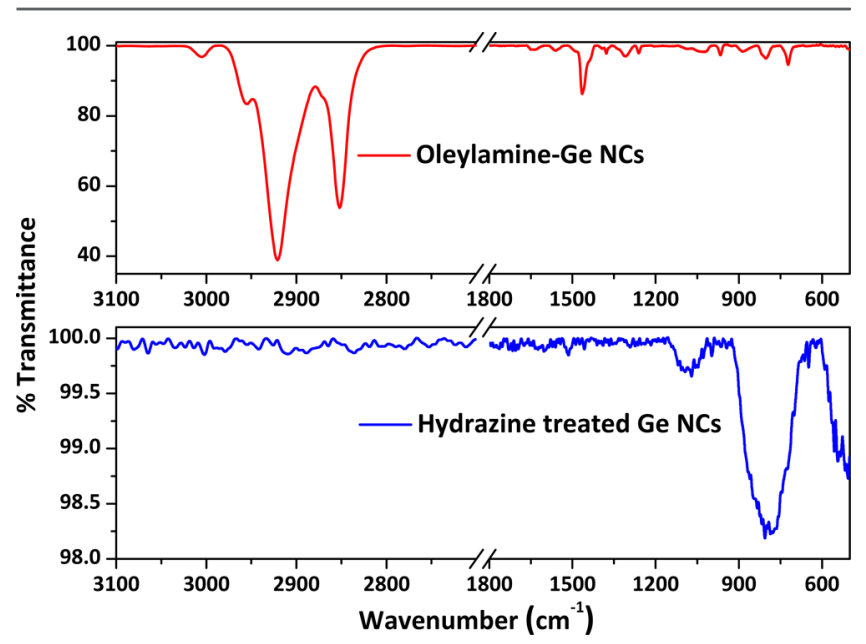

Figure 1. FTIR spectra of oleylamine-capped Ge NCs before and after hydrazine treatment. 
evaporation resulted in a thin film of the NCs on the substrate which was then analyzed on a Bruker D8 Advance diffractometer using the $\mathrm{Cu} \mathrm{K} \alpha$ lines $(40 \mathrm{kV}, 40 \mathrm{~mA})$. The data were acquired with a step size of $0.02^{\circ}$ in the $2 \theta$ range of $20^{\circ}-75^{\circ}$. The data were compared to a powder diffraction file from the International Center of Diffraction Data (ICDD) database (04-0545 diamond cubic Ge). The DebyeScherrer method was used to estimate the crystallite size of the NCs by fitting (pseudo-Voigt) the 111 reflection with Jade 5.0 software.

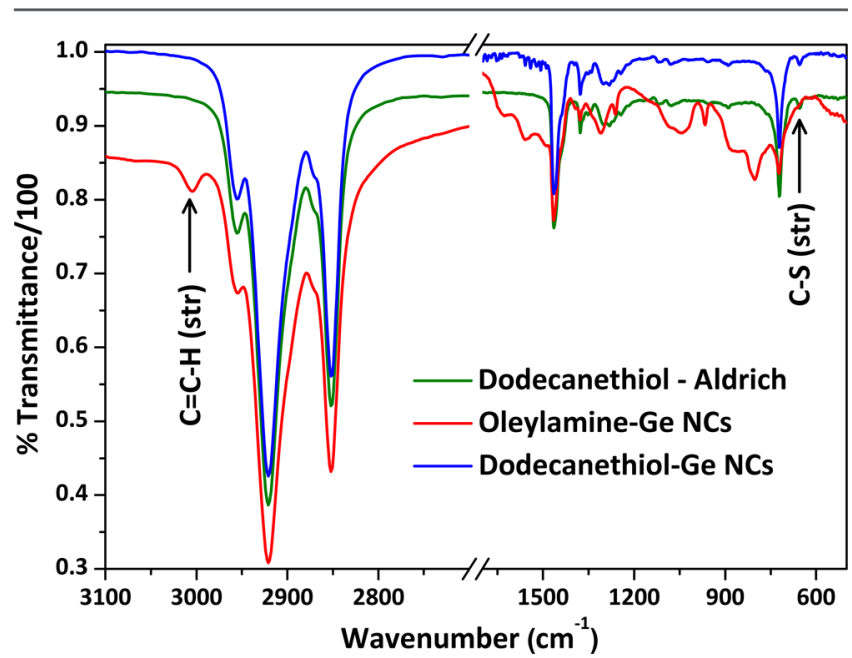

Figure 2. FTIR spectra of neat dodecanethiol, oleylamine-capped Ge NCs, and dodecanethiol-capped Ge NCs.
Samples for transmission electron microscopy were prepared by dropcasting the dispersion onto a carbon-coated copper grid (200 mesh) obtained from SPI. The grids were dried completely before imaging with a JEOL 2500SE electron microscope operated at $200 \mathrm{kV}$. The images were captured using Digital Micrograph software provided by Gatan Inc. Average size and histograms were calculated by measuring the size of individual NCs (100-150 count) using ImageJ software. FTIR measurements were carried out with a Bruker Alpha spectrophotometer. UV-vis-NIR absorption measurements were carried out with a Perkin-Elmer Lambda 750 spectrometer. Ge NCs dispersed in $\mathrm{CDCl}_{3}$ were utilized for solution NMR characterization using a $300 \mathrm{MHz}$ Varian NMR spectrophotometer. The dodecanethiolcapped Ge NCs were heated to dryness under vacuum at $70{ }^{\circ} \mathrm{C}$ for solid-state NMR characterization. ${ }^{1} \mathrm{H}$ MAS NMR experiments were carried out with a Bruker widebore AVANCE $500 \mathrm{MHz}$ spectrometer equipped with an $11.74 \mathrm{~T}$ magnet. The chemical shifts were referenced to TMS.

Samples for surface photovoltage measurements were prepared by the following procedures: $1 \times 1 \mathrm{~cm}^{2}$ Au-coated glass pieces were selected as the conductive substrates and were precleaned by first rinsing with acetone and then soaking in $30 \% \mathrm{H}_{2} \mathrm{O}_{2}$ aqueous solution containing $0.05 \mathrm{M} \mathrm{KOH}$, followed by a final thorough rinse with Milli$Q$ pure water and drying in air. Suspensions of Ge nanoparticles were drop-cast onto $\mathrm{Au}$ substrates and dried in a glovebox. Contact potential difference (CPD) measurements were recorded in vacuum $\left(10^{-7}\right.$ bar) with a vibrating gold mesh reference electrode (Kelvin Probe, $3 \mathrm{~mm}$ in diameter, Delta PHI Besocke) and a Kelvin control 07 (Delta PHI Besocke) with a sensitivity of $1 \mathrm{mV}$. The sample-probe distance was kept consistent (ca. $1 \mathrm{~mm}$ ). A $175 \mathrm{~W}$ Xe arc lamp (PE175-BF) was used as the light source, in conjunction with a

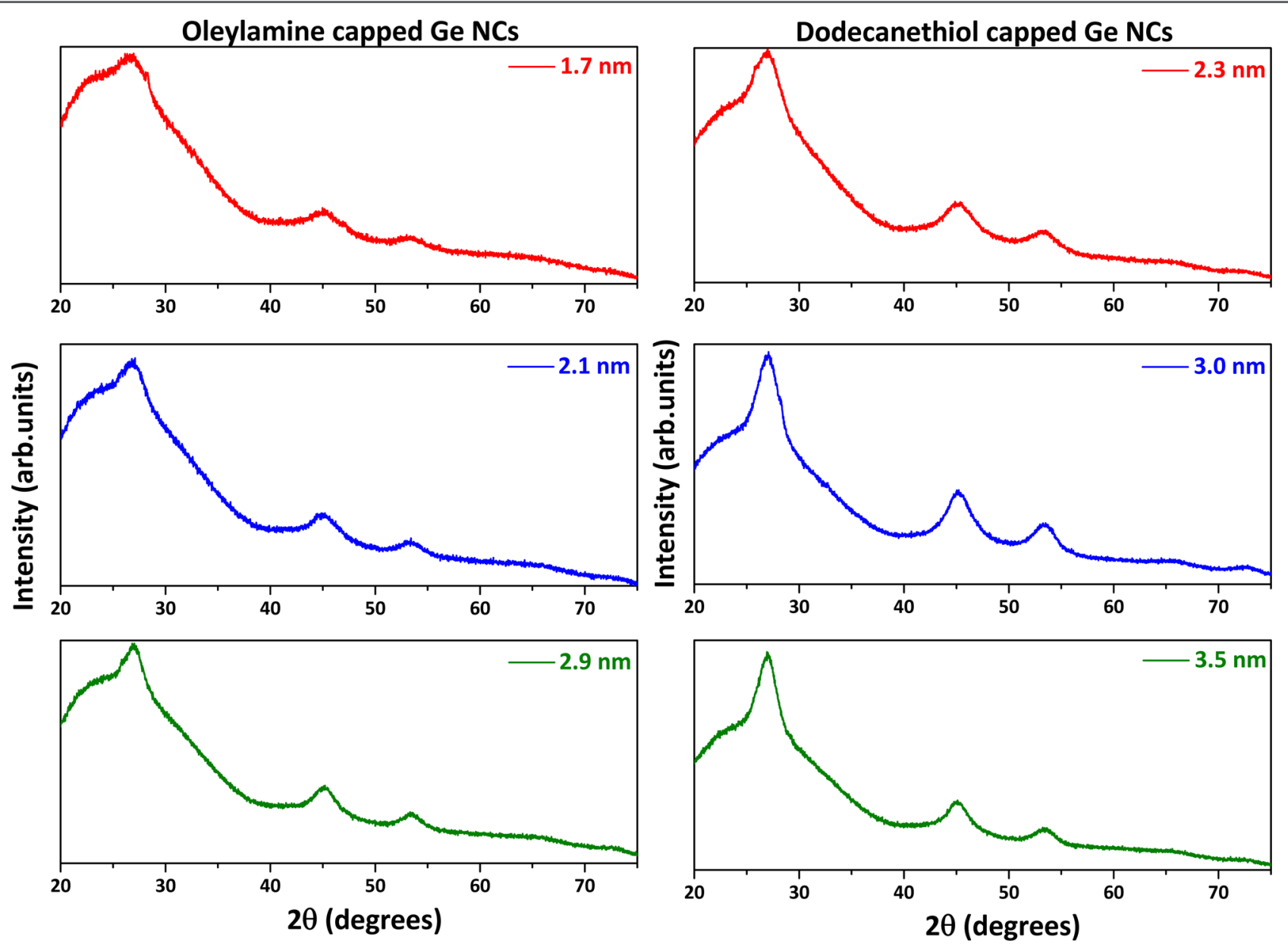

Figure 3. PXRD patterns of oleylamine-capped Ge NCs (left) and dodecanethiol-capped Ge NCs (right). The crystallite size of the NCs obtained from their 111 reflection is provided in the right top corner of each plot. 

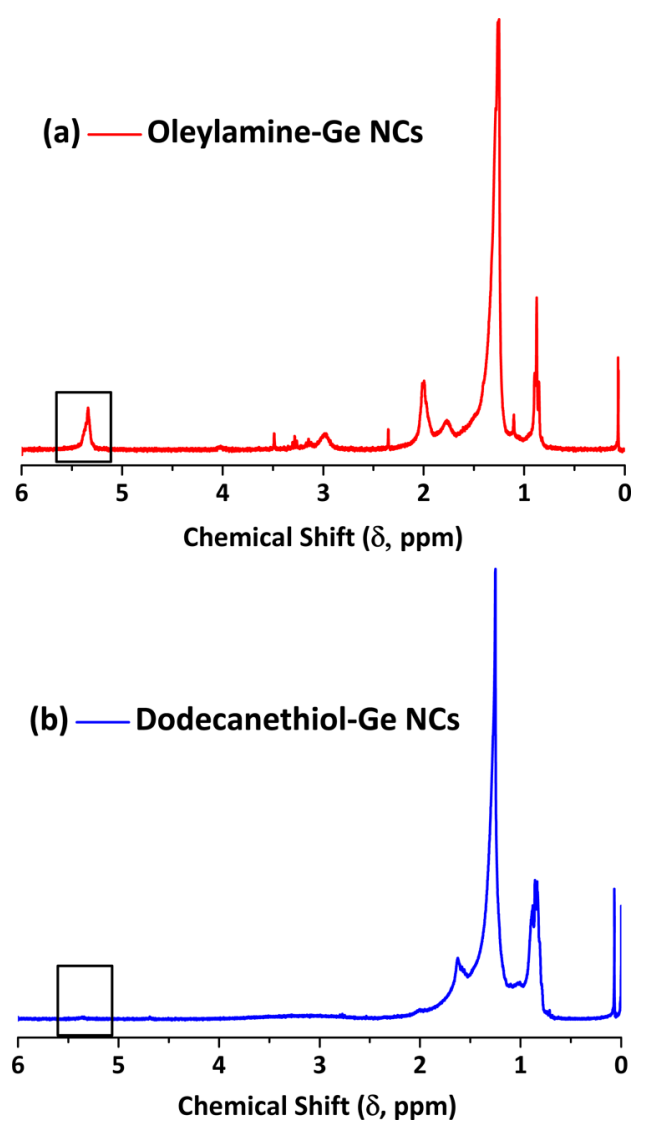

Figure 4. Solution NMR spectra obtained on $\mathrm{CDCl}_{3}$ dispersions of $\mathrm{Ge}$ NCs capped with (a) oleylamine and (b) dodecanethiol. The vinylic protons appear between 5.3 and $5.4 \mathrm{ppm}$ in part a. The full range of the obtained spectra is available in Figure S5 (Supporting Information).

monochromator (Cornerstone 130) to provide monochromatic illumination with an average peak width of $30 \mathrm{~nm}$. SPV spectra were corrected for drift effects due to pressure variations by subtracting a linear background. In the case of a time-dependent SPV measurement, monochromatic illumination at $3.2 \mathrm{eV}(388 \mathrm{~nm})$ was applied. Meanwhile, the CPD signal was monitored over time to probe the photovoltage development upon illumination and decay when illumination was off.

It was observed that the generation of the SPV signal was a sensitive function of the thiol capping reaction conditions. In the case of oil bath heating, a longer reaction time at $80{ }^{\circ} \mathrm{C}(16 \mathrm{~h})$ and a shorter reaction time at $150{ }^{\circ} \mathrm{C}(1 \mathrm{~h})$ were required to generate appreciable photovoltage. Samples prepared with short reaction times at $80{ }^{\circ} \mathrm{C}(6 \mathrm{~h})$ yielded photovoltage less than $50 \mathrm{mV}$, which is comparable to the baseline level. A range of temperatures $\left(85-180^{\circ} \mathrm{C}\right)$ was evaluated with microwave heating for $1 \mathrm{~h}$ with each producing an observable photovoltage.

\section{RESULTS AND DISCUSSION}

Oleylamine-capped Ge NCs were prepared by microwave heating according to the previously reported procedure. ${ }^{14}$ The NC dispersion in hexane or toluene was observed to stay stable for months when stored under an inert atmosphere. However, on exposure to ambient conditions, the NCs lose their dispersibility and slowly precipitate (1-3 days) from the dispersion (Figure S1, Supporting Information). The NCs also lose their dispersibility when subjected to a third purification cycle (dispersion/reprecipitation). In addition, emission characteristics in the expected near-IR region were absent in these NCs. These observations point toward an incomplete surface passivation of the Ge NCs by oleylamine. Long chain thiols have been shown to act as an effective capping ligand for germanium. ${ }^{29,30}$ Resistance to surface oxidation under harsh conditions was also reported for thiol-capped Ge nanowires. ${ }^{29}$ In a recent report, the stability of thiol-capped Ge nanowires as a dispersion in toluene was highlighted. ${ }^{30}$ These observations from the literature provided the incentive to investigate thiolterminated capping ligands to promote the stability of Ge NCs.

The $\mathrm{Ge}-\mathrm{S}$ bond $(551 \mathrm{~kJ} / \mathrm{mol})$ is thermodynamically more stable than the $\mathrm{Ge}-\mathrm{N}$ bond $(257 \mathrm{~kJ} / \mathrm{mol}) .{ }^{33}$ It is possible that the addition of a small quantity of dodecanethiol would result in preferential binding of thiols to the NC surface. However, the addition of dodecanethiol $(1-2 \mathrm{~mL})$ to our reactions resulted in a colorless solution, indicating that the thiols are prohibiting the reduction of germanium iodides under our
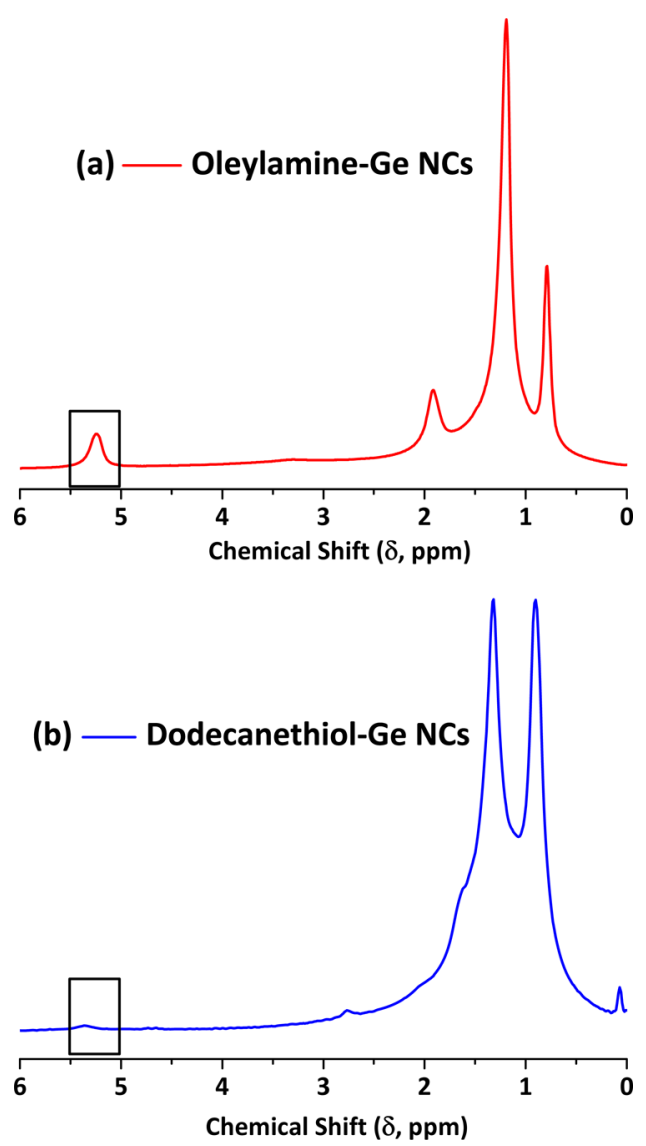

Figure 5. Solid-state NMR spectra obtained on dried Ge NCs capped with (a) oleylamine and (b) dodecanethiol. The vinylic protons appear between 5.3 and $5.4 \mathrm{ppm}$ in part a. The full range of the obtained spectra is available in Figure S5 (Supporting Information).

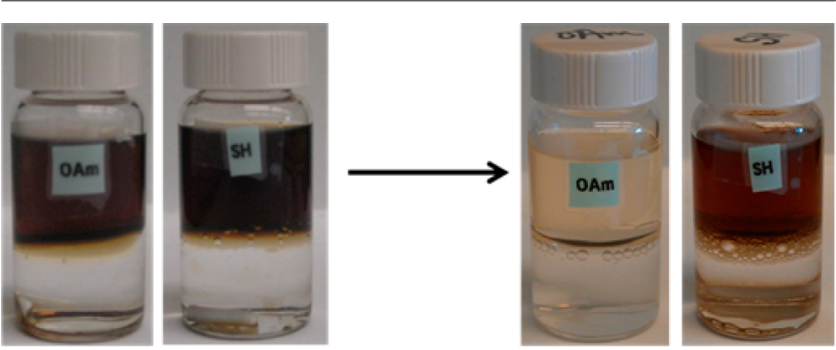

Figure 6. Stability test: oleylamine- and dodecanethiol-capped Ge NC dispersions in toluene stirred with nanopure water for $48 \mathrm{~h}$ under ambient conditions. 

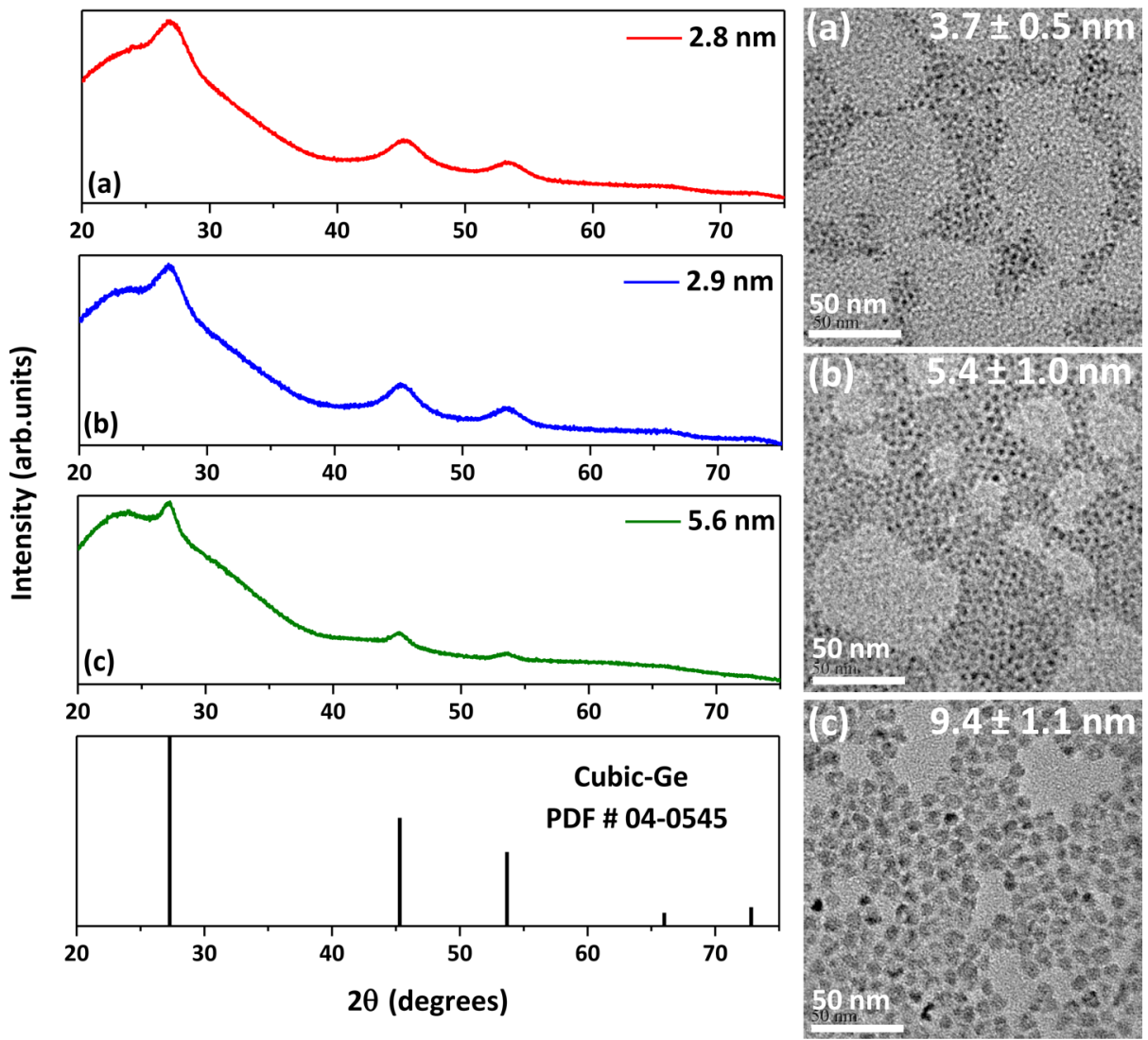

Figure 7. PXRD patterns of dodecanethiol-capped Ge NCs compared to the reference pattern. Crystallite sizes determined from the 111 reflections by the Debye-Scherrer method are provided in the top right corner of the plot. TEM images of the corresponding samples are provided on the right-hand side with their average sizes. Size distribution histograms are provided in Figure S8 (Supporting Information).

operating conditions. A similar observation has been reported in the case of $\mathrm{Ge}$ nanowire synthesis. ${ }^{34}$ Higher reactions temperatures $\left(250\right.$ vs $\left.210{ }^{\circ} \mathrm{C}\right)$ provided identical results. Heating $\left(80^{\circ} \mathrm{C}, 1 \mathrm{~h}\right)$ dodecanethiol with Ge NCs suspended in oleylamine could not establish a complete ligand exchange, as evidenced by the presence of the $-\mathrm{C}=\mathrm{C}-\mathrm{H}$ stretch mode at around $3005 \mathrm{~cm}^{-1}$ (Figure S2, Supporting Information). FTIR and solution NMR experiments indicated a partial ligand exchange when Ge NCs dispersed in toluene were heated with dodecanethiol at RT and as well as $100^{\circ} \mathrm{C}$ (Figures S3 and S4, Supporting Information).

Treatment with dilute solutions of hydrazine was explored to enable complete removal of oleylamine. A number of reports in the literature demonstrate successful removal of long chain ligands on NC surfaces by hydrazine treatment. ${ }^{35-37}$ Toluene dispersions of $\mathrm{Ge} \mathrm{NCs}$ were treated with $5 \mathrm{M}$ hydrazine solution in acetonitrile and stirred at room temperature (RT) for about $1 \mathrm{~h}$. The NCs which previously dispersed in toluene at ease were now observed to flocculate in the same solvent, providing qualitative evidence for modifications in surface coverage. FTIR spectra obtained on the oleylamine-capped NCs before and after hydrazine treatment are given in Figure 1. The absence of characteristic bands corresponding to alkane and alkene $\mathrm{C}-\mathrm{H}$ stretching vibrations in the $2850-3005 \mathrm{~cm}^{-1}$ region confirms the loss of surface coverage. The spectrum was collected in air and the small band around $800 \mathrm{~cm}^{-1}$ (hydrazine treated sample) is assigned to the $\mathrm{Ge}-\mathrm{O}$ stretch due to oxidation of the samples during the time frame of the measurement. Unlike the octadecyl groups bound by a kinetically stable $\mathrm{Ge}-\mathrm{C}$ bond, ${ }^{13}$ oleylamine linked via a labile
$\mathrm{Ge}-\mathrm{N}$ interaction was easily displaced by the mild hydrazine treatment.

Thiol-capping was accomplished by treating the ligandstripped Ge NCs with excess dodecanethiol $(10 \mathrm{~mL})$. The capping reactions were carried out by both oil bath and microwave heating. Oil bath reactions were carried out at $80^{\circ} \mathrm{C}$ (6-16 h). A control experiment was also carried out at $150{ }^{\circ} \mathrm{C}$ $(1 \mathrm{~h})$ with identical result. Much shorter reaction times $(1 \mathrm{~h})$ were needed in the case of microwave heating $\left(85-150{ }^{\circ} \mathrm{C}\right)$. FTIR analysis of the ligand exchanged product revealed the absence of the vinylic protons, indicating a complete ligand exchange. The obtained spectra matched well with spectra obtained on pure dodecanethiol (Figure 2). Additionally, there is no evidence of $\mathrm{Ge}-\mathrm{O}$ stretch in the spectrum. PXRD measurements carried out before and after the ligand exchange confirm a small increase in crystallite size of the Ge NCs, which may be attributed to the additional heating involved in the thiol-capping reaction (Figure 3). No other crystalline impurities are observed. The purification steps (dispersion/ reprecipitation) could now be repeated many times without problems with dispersibility. In addition, vacuum-dried NCs could be subsequently dispersed in toluene. These were the first observations that pointed to an increased stability for the dodecanethiol-capped Ge NCs.

The samples were also analyzed by both solution and solidstate NMR spectroscopy to monitor the ligand exchange. Solution NMR measurements were carried out by dispersing the oleylamine- and dodecanethiol-capped Ge NCs in $\mathrm{CDCl}_{3}$. Similar to the analysis with the FTIR spectra, the peaks corresponding to the vinylic protons were monitored before 
and after the ligand exchange. Vinylic protons appear between 5.3 and $5.4 \mathrm{ppm}$ in oleylamine-capped Ge NCs (Figures 4 and 5 ). Both the solution and solid-state NMR spectra obtained after ligand exchange are characterized by a very small broadening in the region corresponding to vinylic protons, indicating that while undetectable by FTIR the ligand exchange process is not $100 \%$ and that a very small amount of oleylamine remains (Figures 4 and 5).

The stability of the Ge NCs was tested by stirring the toluene dispersions in nanopure water at RT. While the oleylaminecapped Ge NCs oxidized completely within 1 day, the dodecanethiol-capped samples were observed to resist oxidation (Figure 6). UV-vis measurements carried out before and after the water treatment reveal complete loss of absorbance in the case of oleylamine-capped Ge NCs. The dodecanethiol-capped samples retained about $55 \%$ of the original absorbance (Figure S6, Supporting Information). While the thiol ligand resisted oxidation of the Ge nanocrystal surface, the oleylamine-capped Ge NCs were observed to be more susceptible to hydrolysis when in contact with water. No detectable emission was observed in the expected near-IR or in the visible region for dodecanethiol-capped Ge NCs. Similar absence of PL was reported for oleylamine-capped Ge NCs previously, ${ }^{13,14}$ and near-IR emission has only been observed in crystalline Ge NCs the surface of which is capped by $\mathrm{Ge}-\mathrm{C}$ covalent bonds. ${ }^{10,12,25}$

Dodecanethiol-capped Ge NCs of different sizes were prepared and thoroughly characterized by X-ray diffraction, Raman spectroscopy, and electron microscopy before subjecting them to surface photovoltage measurements (see below). PXRD patterns collected on the samples indicated a progressive increase in crystallite size (Figure 7). As observed previously, no other crystalline impurities were observed. Raman measurements on the samples were characterized by the presence of a broad band around $297 \mathrm{~cm}^{-1}$ corresponding to the $\mathrm{Ge}-\mathrm{Ge}$ phonon mode (Figure S7, Supporting Information). The asymmetric broadening of the Raman band with decreasing particle size has been observed in other nanocrystals as well and is attributed to the size effects on the force constants and vibrational amplitudes of the nearest neighbor bonds. ${ }^{38-40}$ TEM images collected on the samples indicate a fairly monodisperse size distribution with average sizes of $3.7 \pm$ $0.5,5.4 \pm 1.0$, and $9.7 \pm 1.1 \mathrm{~nm}$ (Figure 7).

SPV has been shown to be a suitable and powerful technique to measure band gaps of semiconductor powders. ${ }^{41-45}$ The technique is complementary to spectroscopic methods such as diffuse reflectance spectroscopy, ${ }^{46}$ in that can detect the movement of charge carriers generated by super- or sub-band gap absorption. ${ }^{47}$ While the sign of the signal is characteristic of the majority carrier type (electrons or holes), the intensity of the signal provides information about the carrier separation efficiency and carrier concentration. The technique is also known for its sensitivity, as thin films of even one monolayer can provide a meaningful signal. ${ }^{48} \mathrm{~A}$ number of reports have already been published on the successful use of this technique to probe the band gap of nanoscale semiconductors. ${ }^{4-46,49}$

The results of SPV measurements for the three differently sized dodecanethiol-capped Ge NCs are provided in Figure 8a. All three samples prepared by oil bath heating provided a consistent negative CPD signal beginning at $1.25-1.50 \mathrm{eV}$ photon energy and reaching its maximum amplitude of $-0.4 \mathrm{~V}$ at $3.5 \mathrm{eV}$. The negative sign indicates the movement of electrons toward the substrate and the n-type character of the
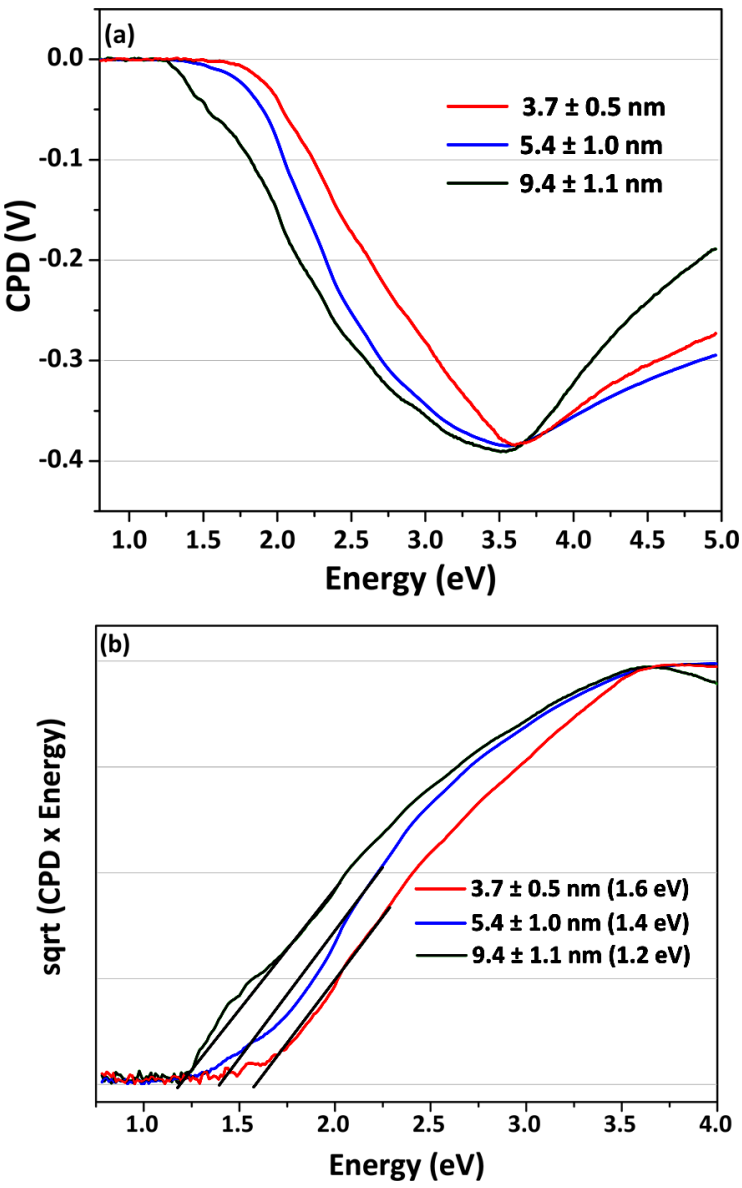

Figure 8. (a) SPV spectra of dodecanethiol-capped Ge NCs of different sizes. (b) Precise onsets of the signals are determined by plotting the square root of $(\mathrm{CPD} \times$ energy) against energy, in the case of an indirect band gap material.

Ge NCs. As can be seen in Figure 8a, the CPD onset exhibits a blue shift with decreasing size, indicating an increase in the band gap with decreasing NC size. To ensure a more accurate determination of the band gaps from the onsets, the extrapolation of $(\mathrm{CPD} \times \text { energy })^{1 / 2}$ versus photon energy is employed to identify the indirect band gap of $\mathrm{Ge}$, as shown in Figure $8 \mathrm{~b} .{ }^{46}$ The extrapolation results in indirect band gap values of 1.6, 1.4, and $1.2 \mathrm{eV}$ for sizes of $3.7,5.4$, and $9.4 \mathrm{~nm}$, respectively. This decrease of the band gap is a direct result of the quantum confinement effect. The estimated band gaps are slightly higher $(0.1-0.2 \mathrm{eV})$ than the values reported for $\mathrm{Ge}$ NCs of comparable sizes reported in the literature. ${ }^{12,14,25}$ In addition to the different capping ligands on the NC surface, these small differences could be attributed to the fact that the band gaps were estimated via different techniques (absorption, emission) previously. However, SPV should provide an accurate measurement of exciton generation in a NC film and be a valuable quantity for applications of these NCs.

In the next set of experiments, we compared oleylamine- and dodecanethiol-capped Ge NCs by SPV (Figure 9). In contrast to the thiol-capped NCs, the oleylamine-capped NCs produce a small negative signal, which is comparable to the baseline level (see also Figure S9, Supporting Information). This suggests an inefficient charge separation in the oleylamine Ge $\mathrm{NC}$ films, possibly due to charge trapping at surface defects. Another possibility could be the bent structure of oleylamine 


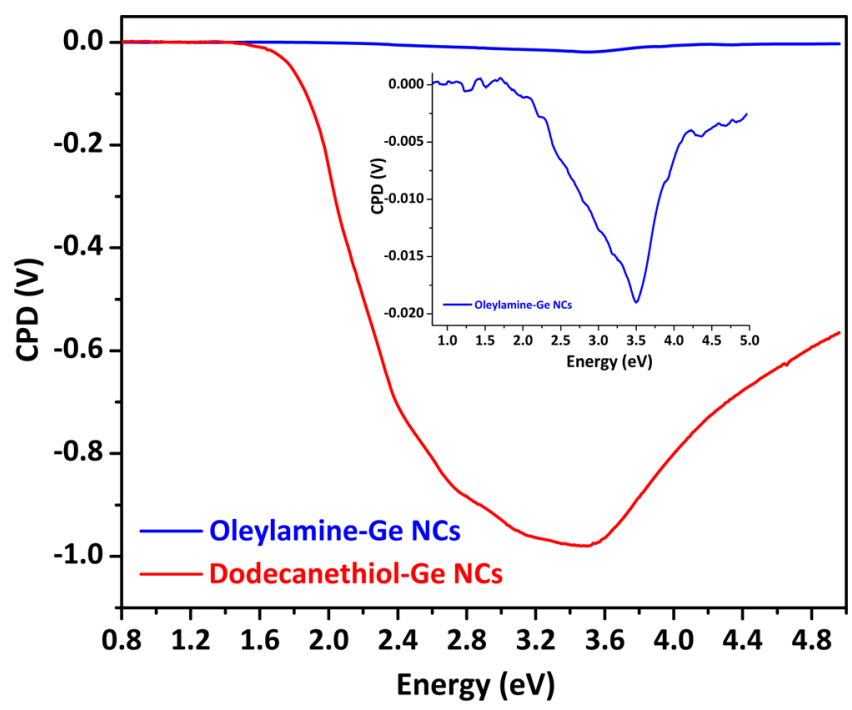

Figure 9. SPV data obtained on dodecanethiol (red)- and oleylamine (blue)-capped Ge NCs of similar size. The inset depicts the expanded version of the SPV spectra obtained on oleylamine-capped Ge NCs.

leading to poor surface coverage and less efficient packing of the Ge NCs in the film. Other than the functional group, a significant difference between oleylamine (18-C) and dodecanethiol (12-C) is their chain length. A thicker ligand shell length may be detrimental for charge transfer between NCs, thus inhibiting electron diffusion to the substrate. Octadecanethiol (18-C) was utilized as a control to ascertain the effect of chain length in the photovoltage generation. As depicted in Figure S10 (Supporting Information), SPV spectrum collected on octadecanethiol-capped Ge NCs exhibits a large negative signal, thereby eliminating the possibility that the shorter chain length was responsible for generation of photovoltage. Thiols are also known to interact favorably with the Au substrate, whereas the amine-Au interaction is relatively weak. To verify that the strong negative CPD from all the thiol-capped Ge samples is not generated due to the $\mathrm{Au}$-thiol dipoles formed at the $\mathrm{Au}-\mathrm{Ge}$ back interface, control measurements were carried out with dodecanethiol deposited on $\mathrm{Au}$, oleylamine deposited on $\mathrm{Au}$, and plain $\mathrm{Au}$ substrate. None of the control measurements led to a significant CPD signal $(\geq 50 \mathrm{mV}$, as shown in Figure S11, Supporting Information), eliminating any possible effects of ligand-substrate interaction. It is also interesting to note that partial exchange of the ligands in the case of samples represented in Figures S3 and S4 (Supporting Information) also lead to SPV signal generation after $34 \mathrm{~h}$ of treatment with dodecanethiol at both RT and $100{ }^{\circ} \mathrm{C}$ by oil bath heating (Figure S12, Supporting Information). This suggests that thiol capping plays an important role in promoting efficient charge separation in these NC films. Systematic thiol substitution and its effect on charge separation has not been studied in this work.

A further investigation on the time-dependent SPV reveals the difference between the oleylamine- and dodecanethiolcapped Ge NCs. Time-dependent measurements were performed under monochromatic illumination $(3.2 \mathrm{eV})$ to ensure band gap excitation of the Ge NCs. The CPD signal was monitored in response to turning the light on and off. As depicted in Figure 10a, the dodecanethiol-capped sample exhibits a large photovoltage signal that saturates after a period of $1000 \mathrm{~s}$ and decays over a period of more than $2000 \mathrm{~s}$ after the light is turned off. On the other hand, the oleylaminecapped Ge sample develops a small negative photovoltage (Figure 10b) within a few seconds upon illumination. The signal decays back to zero within seconds, when the light is
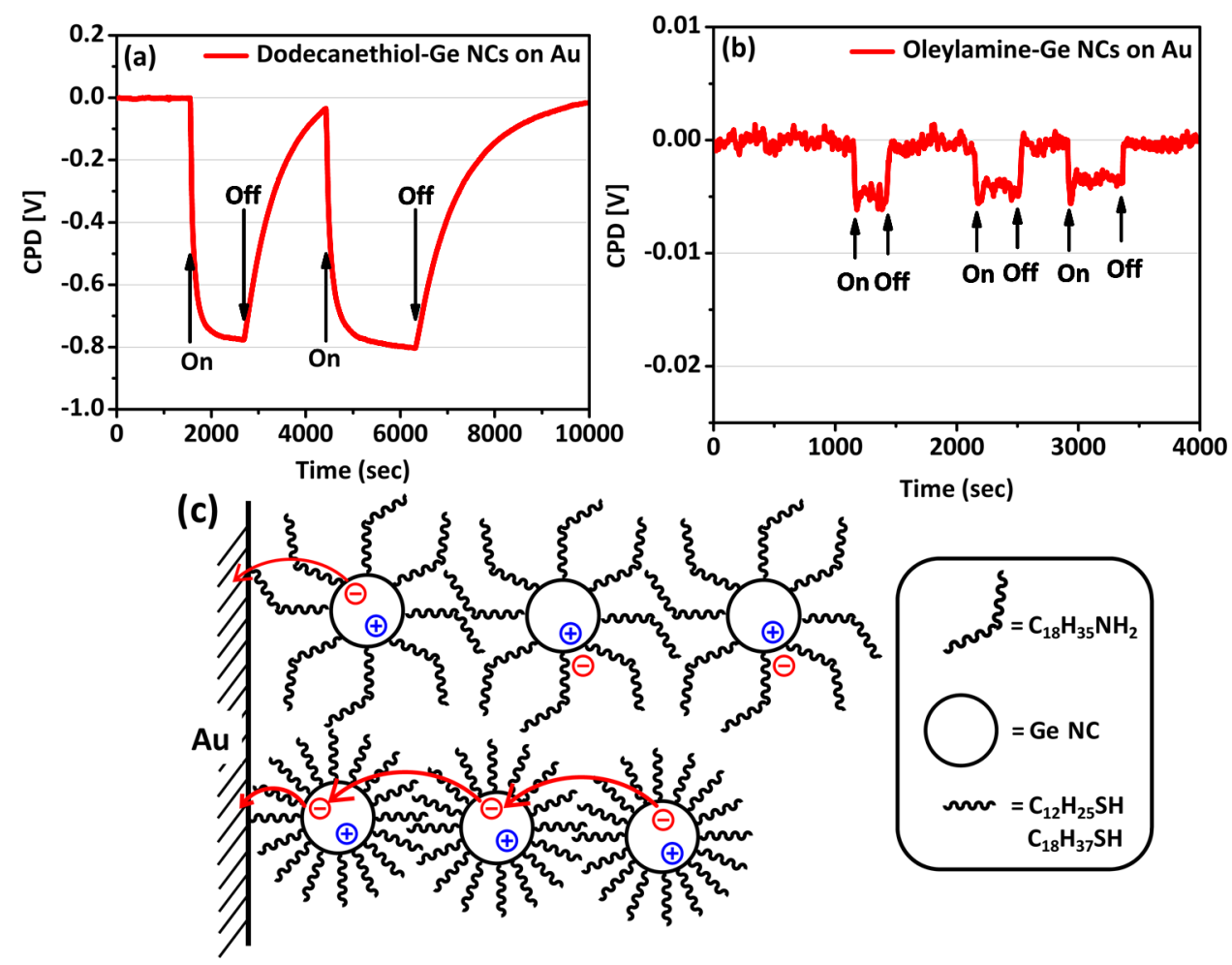

Figure 10. Time-dependent SPV measurements on (a) dodecanethiol-capped Ge NCs and (b) oleylamine-capped Ge NCs. (c) schematic diagram for different charge separation processes in oleylamine- and thiol-capped Ge NCs. 
turned off. These changes are fully reproducible. The variable time scale provides additional support for the charge separation model provided in Figure 10c. Interparticle charge separation in the oleylamine-capped Ge NCs (Figure 10c) is largely inhibited, as a result of the surface trapping from the poorly passivated surface. This leads to a small CPD response and fast saturation/recombination. On the other hand, in the thiolcapped Ge NC film, electrons diffuse through the entire film, causing the larger photovoltage signal and slower saturation. These results highlight the effect of nanocrystal surface defects in modulating photochemical charge separation and transport in NC films.

\section{CONCLUSIONS AND FUTURE OUTLOOK}

In conclusion, we have reported a simple ligand exchange route for the preparation of thiol-capped Ge NCs. The thiol capping was observed to lend a better stability for the Ge NCs than oleylamine. SPV spectroscopy was utilized for the first time to ascertain band gaps as a function of the thiol-capped Ge NC size. As expected, the determined band gaps were observed to blue shift with decreasing size of the NCs. While oleylaminecapped Ge NCs were not responsive for the SPV measurements, dodecanethiol-capped Ge NCs indicated a superior charge separation phenomenon. These results indicate that the thiol-capping could lead to better photovoltaic response for devices made of Ge NC thin films. It also opens up new avenues to explore capping with inorganic sulfur/sulfides, which may lead to Ge NC electronic devices without any organic component in the future.

\section{ASSOCIATED CONTENT}

\section{S Supporting Information}

Figures S1-S12. This material is available free of charge via the Internet at http://pubs.acs.org.

\section{AUTHOR INFORMATION}

\section{Corresponding Author}

*E-mail: smkauzlarich@ucdavis.edu.

\section{Notes}

The authors declare no competing financial interest.

\section{ACKNOWLEDGMENTS}

This work was supported by the National Science Foundation (DMR-1035468 and NSF CBET 933435). We thank Prof. Richard Blair (University of South Florida) for providing phasepure $\mathrm{Ge}$ (II) iodide. We thank Prof. Adam Moule (Chemical Engineering \& Material Science, UC Davis) for providing access to the UV-vis-NIR spectrophotometer. We thank Dr. Ping $\mathrm{Yu}$ from the NMR facility at UC Davis for solid-state NMR measurements. We thank Dr. Alexander Mikhailovsky (Chemistry, UC Santa Barbara) for his help with emission measurements. Marlene M. Amador thanks the Mentorship for Undergraduate Research Participants in Physical and Mathematical Sciences (MURPPS) for funding.

\section{REFERENCES}

(1) Kamat, P. V. Phys. Chem. Lett. 2013, 4, 908.

(2) Mashford, B. S.; Stevenson, M.; Popovic, Z.; Hamilton, C.; Zhou, Z.; Breen, C.; Steckel, J.; Bulovic, V.; Bawendi, M.; Coe-Sullivan, S.; Kazlas, P. T. Nat. Photonics 2013, 7, 407.

(3) Michalet, X.; Pinaud, F. F.; Bentolila, L. A.; Tsay, J. M.; Doose, S.; Li, J. J.; Sundaresan, G.; Wu, A. M.; Gambhir, S. S.; Weiss, S. Science 2005, 307, 538.
(4) Fan, J.; Chu, P. K. Small 2010, 6, 2080.

(5) Vaughn, D. D., II; Schaak, R. E. Chem. Soc. Rev. 2013, 42, 2861.

(6) Depuydt, B.; Theuwis, A.; Romandic, I. Mater. Sci. Semicond. Process. 2006, 9, 437.

(7) Maeda, Y.; Tsukamoto, N.; Yazawa, Y.; Kanemitsu, Y.; Masumoto, Y. Appl. Phys. Lett. 1991, 59, 3168.

(8) Guter, W.; Schöne, J.; Philipps, S. P.; Steiner, M.; Siefer, G.; Wekkeli, A.; Welser, E.; Oliva, E.; Bett, A. W.; Dimroth, F. Appl. Phys. Lett. 2009, 94, 223504.

(9) Lu, X.; Korgel, B. A.; Johnston, K. P. Chem. Mater. 2005, 17, 6479.

(10) Lee, D. C.; Pietryga, J. M.; Robel, I.; Werder, D. J.; Schaller, R. D.; Klimov, V. I. J. Am. Chem. Soc. 2009, 131, 3436.

(11) Vaughn, D. D.; Bondi, J. F.; Schaak, R. E. Chem. Mater. 2010, 22, 6103.

(12) Ruddy, D. A.; Johnson, J. C.; Smith, E. R.; Neale, N. R. ACS Nano 2010, 4, 7459.

(13) Ruddy, D. A.; Erslev, P. T.; Habas, S. E.; Seabold, J. A.; Neale, N. R. Phys. Chem. Lett. 2013, 4, 416.

(14) Muthuswamy, E.; Iskandar, A. S.; Amador, M. M.; Kauzlarich, S. M. Chem. Mater. 2012, 25, 1416.

(15) Sperling, R. A.; Parak, W. J. Philos. Trans. R. Soc. A 2010, 368, 1333.

(16) Heath, J. R.; Shiang, J. J.; Alivisatos, A. P. J. Chem. Phys. 1994, $101,1607$.

(17) Kornowski, A.; Giersig, M.; Vogel, R.; Chemseddine, A.; Weller, H. Adv. Mater. 1993, 5, 634.

(18) Taylor, B. R.; Kauzlarich, S. M.; Lee, H. W. H.; Delgado, G. R. Chem. Mater. 1998, 10, 22.

(19) Taylor, B. R.; Kauzlarich, S. M.; Delgado, G. R.; Lee, H. W. H. Chem. Mater. 1999, 11, 2493.

(20) Ma, X.; Wu, F.; Kauzlarich, S. M. J. Solid State Chem. 2008, 181, 1628.

(21) Tanke, R. S.; Kauzlarich, S. M.; Patten, T. E.; Pettigrew, K. A.; Murphy, D. L.; Thompson, M. E.; Lee, H. W. H. Chem. Mater. 2003, $15,1682$.

(22) Holman, Z. C.; Kortshagen, U. R. Nano Lett. 2011, 11, 2133.

(23) Gerung, H.; Bunge, S. D.; Boyle, T. J.; Brinker, C. J.; Han, S. M. Chem. Commun. 2005, 1914.

(24) Prabakar, S.; Shiohara, A.; Hanada, S.; Fujioka, K.; Yamamoto, K.; Tilley, R. D. Chem. Mater. 2009, 22, 482.

(25) Wheeler, L. M.; Levij, L. M.; Kortshagen, U. R. Phys. Chem. Lett. 2013, 4, 3392.

(26) Henderson, E. J.; Hessel, C. M.; Veinot, J. G. C. J. Am. Chem. Soc. 2008, 130, 3624.

(27) Shirahata, N.; Hirakawa, D.; Masuda, Y.; Sakka, Y. Langmuir 2012, 29, 7401.

(28) Wang, D.; Chang, Y.-L.; Liu, Z.; Dai, H. J. Am. Chem. Soc. 2005, $127,11871$.

(29) Holmberg, V. C.; Korgel, B. A. Chem. Mater. 2010, 22, 3698.

(30) Yuan, F.-W.; Yang, H.-J.; Tuan, H.-Y. ACS Nano 2012, 6, 9932.

(31) Lambert, T. N.; Andrews, N. L.; Gerung, H.; Boyle, T. J.; Oliver, J. M.; Wilson, B. S.; Han, S. M. Small 2007, 3, 691.

(32) Restrepo, D. T.; Lynch, K. E.; Giesler, K.; Kuebler, S. M.; Blair, R. G. Mater. Res. Bull. 2012, 47, 3484.

(33) Luo, Y.-R. In CRC Handbook of Chemistry and Physics, 87th ed.; CRC Press: Boca Raton, FL.

(34) Gerung, H.; Boyle, T. J.; Tribby, L. J.; Bunge, S. D.; Brinker, C. J.; Han, S. M. J. Am. Chem. Soc. 2006, 128, 5244.

(35) Talapin, D. V.; Murray, C. B. Science 2005, 310, 86.

(36) Law, M.; Luther, J. M.; Song, Q.; Hughes, B. K.; Perkins, C. L.; Nozik, A. J. J. Am. Chem. Soc. 2008, 130, 5974.

(37) Church, C. P.; Muthuswamy, E.; Zhai, G.; Kauzlarich, S. M.; Carter, S. A. Appl. Phys. Lett. 2013, 103, 223506.

(38) Xu, C. Y.; Zhang, P. X.; Yan, L. J. Raman Spectrosc. 2001, 32, 862.

(39) Meier, C.; Lüttjohann, S.; Kravets, V. G.; Nienhaus, H.; Lorke, A.; Wiggers, H. Physica E 2006, 32, 155.

(40) Choi, H. C.; Jung, Y. M.; Kim, S. B. Vib. Spectrosc. 2005, 37, 33. 
(41) Kronik, L.; Shapira, Y. Surf. Sci. Rep. 1999, 37, 1.

(42) Kronik, L.; Shapira, Y. Surf. Interface Anal. 2001, 31, 954.

(43) Waller, M. R; Townsend, T. K.; Zhao, J.; Sabio, E. M.; Chamousis, R. L.; Browning, N. D.; Osterloh, F. E. Chem. Mater. 2012, 24, 698.

(44) Frame, F. A.; Townsend, T. K.; Chamousis, R. L.; Sabio, E. M.; Dittrich, T.; Browning, N. D.; Osterloh, F. E. J. Am. Chem. Soc. 2011, 133, 7264.

(45) Zhao, J.; Osterloh, F. E. J. Phys. Chem. Lett. 2014, 5, 782.

(46) Gal, D.; Mastai, Y.; Hodes, G.; Kronik, L. J. Appl. Phys. 1999, 86, 5573.

(47) Fefer, E.; Shapira, Y.; Balberg, I. Appl. Phys. Lett. 1995, 67, 371.

(48) Zillner, E.; Dittrich, T. Phys. Status Solidi RRL 2011, 5, 256.

(49) Kronik, L.; Ashkenasy, N.; Leibovitch, M.; Fefer, E.; Shapira, Y.; Gorer, S.; Hodes, G. J. Electrochem. Soc. 1998, 145, 1748. 